Meta

Journal des traducteurs

Translators' Journal

\title{
Traduire le " huis-clos mental " : étrangeté et discours de la folie dans trois traductions de The Yellow Wallpaper de Charlotte Perkins Gilman
}

\section{Corinne Oster}

Volume 56, numéro 3, septembre 2011

URI : https://id.erudit.org/iderudit/1008329ar

DOI : https://doi.org/10.7202/1008329ar

Aller au sommaire du numéro

Éditeur(s)

Les Presses de l’Université de Montréal

ISSN

0026-0452 (imprimé)

1492-1421 (numérique)

Découvrir la revue

Citer cet article

Oster, C. (2011). Traduire le " huis-clos mental » : étrangeté et discours de la folie dans trois traductions de The Yellow Wallpaper de Charlotte Perkins

Gilman. Meta, 56(3), 493-510. https://doi.org/10.7202/1008329ar
Résumé de l'article

The Yellow Wallpaper, nouvelle autobiographique de Charlotte Perkins Gilman dans laquelle l'auteure dénonce le traitement "médical » de la dépression que subissent les femmes à la fin $\mathrm{du} \mathrm{XIX}^{\mathrm{e}}$ siècle aux États-Unis, a fait l'objet de trois traductions en français en 1976, 1982 et 2002, quatre-vingts ans après sa parution. Le présent article étudie la manière dont ces trois traductions successives mettent en valeur ou négligent le discours féministe de l'auteur, ainsi que la manière dont le discours de la folie, d'un point de vue stylistique, est traduit dans les trois versions francophones de la nouvelle. 


\title{
Traduire le « huis-clos mental »: étrangeté et discours de la folie dans trois traductions de The Yellow Wallpaper de Charlotte Perkins Gilman
}

\author{
CORINNE OSTER \\ Université Charles-de-Gaulle Lille 3, Villeneuve d'Ascq, France \\ corinne.oster@univ-lille3.fr
}

\begin{abstract}
RÉSUMÉ
The Yellow Wallpaper, nouvelle autobiographique de Charlotte Perkins Gilman dans laquelle l'auteure dénonce le traitement «médical» de la dépression que subissent les femmes à la fin du xIx ${ }^{\mathrm{e}}$ siècle aux États-Unis, a fait l'objet de trois traductions en français en 1976, 1982 et 2002, quatre-vingts ans après sa parution. Le présent article étudie la manière dont ces trois traductions successives mettent en valeur ou négligent le discours féministe de l'auteur, ainsi que la manière dont le discours de la folie, d'un point de vue stylistique, est traduit dans les trois versions francophones de la nouvelle.
\end{abstract}

\begin{abstract}
In The Yellow Wallpaper, Charlotte Perkins Gilman's autobiographical short story, the author shows how women, at the end of the nineteenth century, suffered from the "medical" treatment they were subjected to in order to cure depression. Eighty years after its original publication, the story was translated into French in 1976, 1982 and 2002. This article examines the ways in which these three translations highlight or neglect the author's feminist discourse, and looks at the stylistic rendering of the discourse of madness in the three francophone versions of the story.
\end{abstract}

\section{MOTS-CLÉS/KEYWORDS}

féminisme, folie, traduction de nouvelle, retraduction, littérature féminine feminism, madness, short story translation, retranslation, women's literature

\section{Introduction}

Le présent article a pour objet l'examen des trois traductions successives de la nouvelle autobiographique de Charlotte Perkins Gilman (1860-1935), The Yellow Wallpaper, dans laquelle l'auteur dénonce le traitement «médical» de la dépression que subissent les femmes à la fin du XIx ${ }^{\mathrm{e}}$ siècle aux États-Unis. L'analyse comparative des différences de traduction entre chacun de ces textes nous permettra de mettre en évidence les diverses formes que prend le discours féministe de l'auteur en traduction, ainsi que la manière dont le discours de la folie est appréhendé dans ce contexte.

The Yellow Wallpaper, publié en $1892^{1}$, est rédigé sous la forme d'un journal intime écrit par une jeune femme souffrant de dépression, cloîtrée dans sa chambre par un mari bien-pensant et des docteurs qui, forts d'un savoir scientifique irréfutable, la condamnent à un traitement basé sur une inactivité totale qui va la rendre progressivement folle: elle devient obsédée par le papier peint de la chambre, où elle voit bientôt une femme prisonnière de barreaux à laquelle elle s'identifie avant d'arracher tout ce papier pour tenter de la délivrer. Dans cette nouvelle, l'auteure 
entend dénoncer le traitement "médical» dont elle fut elle-même l'objet - ou plus exactement la victime - et qui consista, à la suite d'une dépression postnatale, à suivre une cure de sommeil prescrite par un des plus grands cliniciens de l'époque: Weir Mitchell, qui recommandait à ses patientes un repos absolu pendant toute la durée du traitement, et qui offrira même à l'auteure les précisions suivantes: «live as domestic a life as possible, [...] have but two hours intellectual life a day, [...] and never ... touch pen, brush or pencil for as long as [you live]» (Gilman 1913) - conseils qui auront pour unique effet d'aggraver ses symptômes. Comme l'inaction ne sied point à Gilman, sa guérison ne viendra que de son retour au travail d'écriture, qui donnera naissance au "Yellow Wallpaper", nouvelle retraçant son calvaire.

Lors de sa parution, The Yellow Wallpaper, bien que reconnu alors comme l'œuvre d'une importante féministe et réformatrice sociale, est surtout commenté par les critiques comme conte d'horreur gothique ou histoire de fantômes (genres à la mode) avant de tomber dans l'oubli pendant près d'un siècle ${ }^{2}$. La nouvelle est redécouverte au début des années 1970 grâce à l'essor du mouvement féministe, et les presses féministes américaines (Feminist Press, Old Westbury, NY) en publient une réédition en 1973, agrémentée d'une postface d'Elaine R. Hedges qui fera de cette œuvre un best-seller. Cette réédition à succès entraîne alors ses premières traductions en langue étrangère. Après avoir fait frissonner ses lecteurs et auditeurs d'antan, The Yellow Wallpaper devient alors dans les années 1970 un texte emblématique de la condition des femmes et de leur lutte pour échapper au joug de l'autorité patriarcale. De nouvelles lectures de la nouvelle commencent à abonder, tant en Europe qu'en Amérique du Nord, et il est même avancé que ces interprétations ne peuvent être élaborées que parce que les lecteurs de la fin du $\mathrm{xx}^{\mathrm{e}}$ siècle ont accès à des outils critiques, féministes ou psychanalytiques, qui n'étaient pas à leur disposition quatre-vingts ans auparavant (Golden 2004: 74).

\section{Contexte et enjeux théoriques}

C'est dans ce contexte de la réédition féministe du texte de Gilman que paraissent deux des traductions examinées ici (1976 en France ${ }^{3}$ et 1982 au Québec ${ }^{4}$ ), tandis que la troisième traduction est publiée en $2002^{5}$, plus de trente ans après la redécouverte de la nouvelle. Notons que les trois traductions de cette nouvelle en français ont été faites par des femmes.

La première traduction, sobrement traduite sous le titre: Le papier peint jaune, date de 1976 et est l'œuvre du collectif de traduction des éditions Des femmes ${ }^{6}$, maison d'édition fondée par le groupe Psychanalyse et Politique d'Antoinette Fouque, également co-fondatrice du Mouvement de libération des femmes (MLF) quelques années auparavant, et dont la vocation était de «rendre visible l'apport des femmes à tous les champs de la connaissance, de la pensée et de l'action» (Fouque s.d. ${ }^{7}$ ). Les éditions Des femmes étaient dans les années 1970 la première maison d'édition de ce type en Europe, et dans un contexte relativement timide, la publication de la nouvelle de Gilman devait ainsi s'insérer comme ouvre de littérature militante sans être nécessairement féministe. L'ouverture des éditions Des femmes à «toutes les démarches de lutte, luttes individuelles ou collectives, et dans quelque champ que ce soit» (Fouque s.d.) s'explique en partie par l'idéologie universaliste française, mais n'enlève rien au caractère ouvertement militant dans lequel la nouvelle va être publiée. La 
traduction, sous la forme d'un petit livre de cinquante pages, est agrémentée d'une courte page d'introduction essentiellement biographique dans laquelle Gilman est présentée comme une auteure «consciente des injustices infligées aux femmes» et qui «ne cesse de militer à travers les États-Unis et l'Europe, pour le socialisme et les droits des femmes» (Gilman 1976: 7). La traduction des éditions Des femmes entend donc placer le texte de Gilman dans le contexte spécifique désigné par Hedges dans son édition de 1973 et en propose ensuite une traduction brute sans offrir d'analyse qui guiderait sa lecture.

La deuxième traduction de The Yellow Wallpaper paraît au Québec en 1982. Intitulé La chambre au papier peint, ce petit livre de 56 pages est publié par Naaman, maison d'édition qui à cette époque publie nombre d'œuvres de littérature francophone (Antilles, Afrique, pays arabes...) féministe et de poésie. La traduction est signée Mary M. Millman, alors professeure de français à Auburn University (Alabama, États-Unis), et qui travaille à l'époque sur les périodiques féminins québécois. Cette traduction est accompagnée d'une postface d'Elaine R. Hedges, qui on l'a vu avait fait redécouvrir The Yellow Wallpaper aux États-Unis en 1973. Cette postface assez fournie, traduction de la postface originale de Hedges, présente l'auteure, son œuvre, et est assortie d'une analyse du texte et d'un commentaire sur le féminisme. Le texte traduit est précédé de la dédicace: «C’est à toute cette classe de femmes vaincues et même détruites, à cette masse de talents ratés ou à demi-ratés qu'est dédié le présent récit».

Si la France des années 1970 et 1980 ne développe pas de théorie particulière relative au féminisme en traduction, le Canada, quant à lui, s'inscrit dans une situation spécifique de par son bilinguisme officiel et la traduction, de ce fait, devient un terrain de réflexion et d'expérimentation. C'est ainsi que fleurissent à partir du milieu des années 1970 nombre de théories de la traduction menées par des intellectuelles et écrivaines influentes (Nicole Brossard, Barbara Godard) : ces pionnières des théories de la traduction féministe y postulent que le traducteur - ou plutôt la traductrice - doit affirmer sa visibilité dans le processus de traduction. Dans un article publié sur la traduction féministe au Québec, Von Flotow (1991) identifie plusieurs pratiques utilisées par les traductrices féministes pour rendre plus visible leur travail de traduction et le processus de création lui-même, afin de l'opposer aux normes d'écriture et de publication patriarcales traditionnelles. Les traductrices qu'elle étudie ont ainsi recours à des stratégies spécifiques dont Von Flotow donne quelques exemples: «supplementing»: intervention de la traductrice dans le texte original afin de spécifier certains intraduisibles principalement liés au genre et à sa politique; "prefacing and footnoting» : intervention dans le paratexte: préface, postface, notes de bas de page...; et «hijacking»: intervention féministe de la traductrice dans un texte qui ne l'est pas nécessairement et appropriation explicite du texte traduit (Von Flotow 1991: 74-79).

La traductrice de l'édition québécoise de 1982 ne semble pas appartenir à ce mouvement, ne serait-ce que de par la nature de la traduction produite (dont nous parlons en détail un peu plus bas), et elle marque brièvement sa présence sur la quatrième de couverture ${ }^{8}$, avant de déléguer la parole à Elaine R. Hedges dans la postface (qu'elle a probablement traduite elle-même). Aucune intervention de ce type ne se retrouve dans l'édition française de 1976, simplement présentée par une courte page d'introduction et une quatrième de couverture citant quelques extraits du texte. Le 
marquage de ce dernier comme texte féministe s'effectue néanmoins par le biais du paratexte, aussi court soit-il. L'édition québécoise quant à elle offre à ses lecteurs non seulement une présentation féministe du texte de Gilman, mais en donne aussi une grille de lecture précise grâce à la postface de Hedges, laquelle joue alors son rôle didactique (Simon 1996: 14). Les traductrices des deux éditions, en revanche, ne semblent pas intervenir explicitement dans le texte lui-même.

La traduction quant à elle - en tant que réécriture à part entière - répond également à des impératifs d'acclimatation, car comme le souligne André Lefevere, elle s'inscrit dans un dispositif idéologique spécifique: "The non-professional reader increasingly does not read literature as written by its writers, but as written by its rewriters» (Lefevere 1992: 4). L'accès au texte se fait donc nécessairement par le filtre idéologique dominant. Mais avant d'aborder la troisième traduction de la nouvelle de Gilman, un autre facteur doit être mentionné, qui complexifie l'appréhension des trois publications. La traduction de 2002 est en effet une retraduction, détail dont on peut dire à la lecture des travaux d'Antoine Berman et de Paul Bensimon qu'il a son importance. Ce dernier note en effet que les premières traductions sont principalement des introductions, tandis que les retraductions répondent à d'autres impératifs :

La première traduction procède souvent - a souvent procédé - à une naturalisation de l'œuvre étrangère; elle tend à réduire l'altérité de cette œuvre afin de mieux l'intégrer à une culture autre. [Elle] vise généralement à acclimater l'œuvre étrangère en la soumettant à des impératifs socio-culturels qui privilégient le destinataire de l'œuvre traduite. (Bensimon 1990: ix)

On peut avancer que le texte de Gilman paru en France en 1976 fait alors partie d'un corpus d'œuvres inédites en français présentées sous l'angle spécifique du féminisme et qu'il doit s'intégrer au mieux dans le paysage idéologique français, tandis que celui de 2002 arrive, pour ainsi dire, en terrain conquis. Il faut cependant se demander si la traduction québécoise de 1982 doit être considérée comme une retraduction, ou une autre "première traduction». Antoine Berman considère que toute traduction entre dans l'espace de la retraduction si l'auteur a déjà été traduit, la retraduction étant pour lui une opération qui a lieu quand un premier «état donné de la langue, de la littérature, de la culture» ne répond plus à son "rôle de révélation et de communication des œuvres» (Berman 1990 : 3). Les deux contextes institutionnels français et québécois sont-ils assez liés pour justifier la qualification de la traduction de 1982 en tant que retraduction? La réponse, si l’on s'en tient aux préceptes énoncés par Bensimon, semble se trouver dans le texte lui-même. En effet, si l'on regarde les deux textes traduits, il saute aux yeux que le texte de 1982 est le plus cibliste des deux (voir micro-analyse ci-dessous). Or, Bensimon note dans son article que les retraductions diffèrent fondamentalement des premières traductions car

[1]a première traduction ayant déjà introduit l'œuvre étrangère, le retraducteur ne cherche plus à atténuer la distance entre les deux cultures; il ne refuse pas le dépaysement culturel: mieux, il s'efforce de le créer. Après le laps de temps plus ou moins grand qui s'est écoulé depuis la traduction initiale, le lecteur se trouve à même de recevoir, de percevoir l'œuvre dans son irréductible étrangeté, son « exotisme». La retraduction est généralement plus attentive que la traduction-introduction, que la traduction-acclimatation, à la lettre du texte source, à son relief linguistique et stylistique, à sa singularité. (Bensimon 1990: ix-x) 
Les deux traductions de 1976 et 1982 peuvent donc être considérées comme simultanées, naissant et s'inscrivant dans deux continents et deux contextes institutionnels différents.

La dernière traduction de The Yellow Wallpaper paraît en France en 2002 sous le titre de La séquestrée. Elle est éditée par Phébus, maison d'édition qui dans sa préface au livre signale que si le texte «est sans doute familier à ceux qu'intéresse l'histoire des idées féminines », il «devrait l'être à l'oreille de tous ceux qui aiment la littérature tout simplement - et singulièrement celle qui se fonde moins sur les idées que sur telle révolte centrale de l'esprit».

On le voit, la politique éditoriale annoncée de cette retraduction (l'original des éditions Des femmes est mentionné dans la note de l'éditeur) se veut inclusive, généraliste. La justification annoncée pour la nouvelle traduction quant à elle est celle de la traductrice, Diane de Margerie, auteure bien connue de romans et d'essais, et membre du jury du prix Fémina, "qui porte une affection particulière - et ancienne - à ce texte, [et qui] a tenu à le faire exister dans une traduction nouvelle où elle a mis autant d'engagement intime que de fidélité» (Gilman 2002: 9-10). On note dans cette édition l'intervention personnelle de la traductrice à la fin de la nouvelle, dans une postface de trente pages où Diane de Margerie présente le texte à l'intérieur d'une biographie de l'auteure situant cette dernière dans le contexte littéraire de l'Amérique $\mathrm{du} \mathrm{XIX}^{\mathrm{e}}$ siècle où l'indépendance de la femme était un combat de tous les instants, et où la contribution de Gilman au féminisme apparaît clairement.

La question qui se pose alors est la suivante: pourquoi, en 2002, retraduire le texte de 1976? Certes, le livre publié aux éditions Des femmes n'a en 2002 pas encore été réédité (il ne l'est que depuis 2007), mais suivant Berman, on peut avancer une autre hypothèse selon laquelle, dans la mesure où toute traduction est nécessairement défaillante, toute retraduction «surgit de la nécessité [...] de réduire la défaillance originelle» (Berman 1990: 2). On peut ainsi se demander dans quelle mesure cette dernière traduction répond à des impératifs de correction. Il apparaît nécessaire, pour répondre pleinement à cette question - et d'autres, d'examiner le texte des trois traductions en détail, afin de voir dans quelle mesure chaque traduction met en valeur le message féministe de la nouvelle.

\section{Le texte}

La traduction de la nouvelle de Gilman pose d'emblée plusieurs problèmes d'ordre général qui méritent d'être signalés. Rappelons tout d'abord que ce texte, publié en 1892, n'est traduit en français pour la première fois que quatre-vingts ans après sa parution. Les écarts entre langue source et cible sont donc de deux natures: d'une part, la traductrice transpose la nouvelle dans une langue-culture et un lieu différents, mais d'autre part, l'écart temporel pose la question de l'historicité: faut-il le transposer dans une langue plus contemporaine afin de «supprimer la distance de l'historicité linguistique», ou préserver le style un peu désuet de la nouvelle et ainsi «se condamner à l'archaïsme artificiel»(Genette 1982: 297)? Ces choix, rappelons-le, seront également conditionnés par le statut de traduction ou de retraduction conféré à chacun des textes.

La manière dont va s'appréhender l'étrangeté du texte dépend ainsi également de ces choix de traduction préliminaires. Mais avant toute micro-analyse, nous pouvons 
déjà dégager quelques tendances: la traduction de 1976 et celle de 2002 semblent avoir pris le parti d'une écriture plus proche de l'étrangeté du texte original que celle de 1982, dont la lecture semble plus fluide. Après avoir établi que la traduction de 1982 constitue une deuxième traduction originale, on pourra se demander si la retraduction de 2002 présente les caractéristiques sourcières identifiées par Berman, et enfin, naturellement, dans quelle mesure ces traductions répondent ou non au discours féministe véhiculé par le texte.

En ce qui concerne la micro-analyse du texte, il serait présomptueux de vouloir traiter de la traduction de toutes les «étrangetés» que recèle le texte original, nous nous concentrerons donc sur les occurrences les plus significatives.

Le journal intime de la narratrice est un texte assez brut, construit autour du non-dit, et (vingt ans avant Freud) à la psychologie quasi inexistante, qui témoigne avant tout des deux pulsions contradictoires d'une femme qui veut guérir en faisant confiance à son mari d'une part, mais qui d'autre part se rend bien compte, même si c'est à mots couverts, qu'elle est prisonnière de son autorité (en tant que mari et médecin) et qu'elle n'ira mieux qu'en le trahissant. L'étrangeté du texte, longtemps interprétée à travers le prisme d'une lecture fantastique, mais qui est bien une écriture «de la folie» en réaction aux canons de l'époque, est alors signifiée par quatre éléments principaux:

- la mise en page du texte;

- la syntaxe défaillante;

- les oxymores et déclarations contradictoires qui émaillent le récit;

- les répétitions obsessionnelles de certains mots ou de certaines idées.

\subsection{La mise en page du texte}

Le texte original est composé de paragraphes très courts, parfois d'une à deux lignes, qui viennent renforcer l'idée de fragmentation des pensées de la narratrice. Cette mise en page est globalement respectée dans les trois traductions, mais on note l'espacement des paragraphes dans la version de 1976, qui insiste sur cette fragmentation, et donne ainsi une sensation d'ellipse et de non-dit plus marqués que dans les deux autres traductions. Ces espacements (dont certains existent dans l'original pour marquer le passage du temps) disparaissent assez systématiquement dans la traduction de 1982, qui paraît donc plus fluide mais semble passer à côté d'une mise en forme signifiante. Elle relève également d'un style beaucoup plus écrit, comme nous le verrons ultérieurement. La traduction de 2002, quant à elle, respecte assez scrupuleusement la mise en page originale, à l'exception de quelques rares occurrences. En l'absence d'information complémentaire, il est difficile de dire si l'espacement des paragraphes est le fruit d'un choix délibéré des traductrices ou s'il a été décidé par leur maison d'édition respective, mais l'édition de 1976 semble présenter la mise en page la plus audacieuse des trois (figure 1). 


\section{FIGURE 1}

format des trois traductions de The Yellow Wallpaper: (1) Le papier peint jaune, 1976; (2) La chambre au papier peint, 1982; (3) La séquestrée, 2002

(1)

Je ne me suis occupée de rien naturellement. C'est Jennie qui s'occupe de tout à présent.

Mais je suis tout de même fatiguée.

John dit que si je ne me rétablis pas plus rapidement, il m'enverra chez Weir Mitchell à l'automne.

Mais je n'ai aucune envie d'y aller. Une de mes amies l'a consulté et elle dit qu'il est tout à fait comme John et comme mon frère, et même cent fois pire !

Et c'est une telle entreprise d'aller si loin!

$\checkmark$ 'ai le sentiment qu'il est inutile de faire le moindre geste et je deviens terriblement irritable et chagrine.

Je pleure pour un rien, je pleure presque toute la journée. Pas en présence de John bien sûr, ni de personne d'ailleurs, seulement quand je suis seule.

Et je suis souvent seule en ce moment.

John est très fréquemment retenu en ville pour des urgences, et Jennie est si gentille qu'elle me laisse seule quand je le lui demande.

\section{2}

(2)

Voilà la sœur qui monte l'escalier!

Ainsi, le 4 juillet est done passé. Les gens sont tous partis et je suis épuisée. John a pensé que cela pourrait me faire du bien d'avoir de la compagnie, alors nous avons invité seulement Maman, Nellie et les enfants à passer un week-end.

Bien sûr, je n'ai rien fait. Jennie s'occupe de tout maintenant.

Mais j'étais quand même fatiguéc.

John dit que si je ne me remets pas plus vite, il m'enverra chez Weir Mitchell à l'automne.

Mais je ne veux pas du tout $y$ aller. Une fois, il a pris en charge une de mes amies qui m'a dit qu'il était tout à fait comme John et mon frère, et même pire!

D'ailleurs, c'est toute une affaire que de voyager si loin.

Ce n'est même pas la peine de bouger le petit doigt, je deCe n'est meme pas la peine de bouger
viens terriblement irritable et maussade.

Je pleure la plupart du temps, et pour un rien.

Naturellement, je me retiens quand John ou n'importe qui d'autre est là, je me laisse aller seulement quand je suis seule.

Et je le suis souvent ces jours-ci. John est fréquemment retenu en ville par des appels urgents, et Jennie est gentille, elle me laisse tranquille quand je le veux.

Alors, je me promène un peu dans le jardin ou le long de ce ravissant chemin que j'ai décrit auparavant; je m'assieds ce ravissant chemin que j'ai décrit auparavant; je m'assieds
dans la véranda entourte de roses, et je passe beaucoup de temps au lit.

Je commence à aimer vraiment cette chambre, malgrt son papier. Peut-être précisement à cause du papier.

11 m'obsède tellement!

Je me couche sur cet immense lit fixé au sol par des clous. je pense et je suis des yeux le dessin pendant des heures.

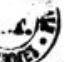

La Chambre au papier peint/17

(3)

\section{LA SEQQUESTRÉE}

Je n'arrive pas à penser que je puisse me rendre utile à quoi que ce soit, et je deviens terriblement irritable et morose.

Un rien me fait pleurer, et je pleure la plupart du temps. Bien sûr, je m'abstiens quand John est là, ou n'importe qui d'autre : je pleure quand je suis seule.

Et maintenant je suis seule très souvent, John étant fréquemment retenu en ville par ses patients. Jennie est compréhensive : elle me laisse tranquille quand j'en ai envie.

Alors je me promène un peu dans le jardin, je descends la belle allée; je m'assieds sous le porche couvert de roses mais je reste aussi très souvent couchée, ici, dans cette chambre du haut.

Vraiment, je m'affectionne à cette chambre malgré le papier peint. $A$ cause de lui, peutêtre?

Tant il m'obsède.

\section{LA SÊQUESTREE}

Je reste étendue sur ce grand lit inamovible (je crois qu'il est fixé au sol par des clous) et, heure après heure, je fais le tour du motif sur le mur. C'est un véritable exploit, je vous assure. Je commence, disons, par le bas, là dans le coin encore intact, et je décide pour la millième fois que je suivrai ce motif démentiel jusqu'au bout pour parvenir à une sorte de conclusion.

Je connais quelque peu les principes du dessin et je vois bien que ce motif ne repose sur aucune loi de circularité, d'alternance, de répétition ou de symétrie, ni sur aucun système connu.

Il ne sait que se répéter sur chaque lé.

Vu d'un certain angle, chaque lé est indépendant; les courbes floues et les arabesques d'une sorte de dessin romantique décadent, atteint de delirum tremens, se fondent de haut en bas, de bas en haut. en colonnes d'une agressivité solitaire. Sous un autre angle, elles se rencontrent en diagonale et leurs contours se perdent en grandes vagues étendues. penchées - vision d'horreur - 


\subsection{La syntaxe défaillante}

La nouvelle de Gilman se caractérise par une tendance à rédiger des paragraphes courts, mais aussi parfois des phrases courtes, voire sans verbe. Bien que ces phrases tronquées ne constituent nullement l'essentiel du texte, elles ont leur importance: d'un point de vue stylistique, elles illustrent elles aussi à leur manière la fragmentation des idées de la narratrice; elles sont par ailleurs à rapprocher des stratégies d'écriture féministes encore relativement inédites à la fin du XIX ${ }^{\mathrm{e}}$ siècle et qui vont à l'encontre du canon littéraire traditionnel. Les occurrences sont répertoriées ci-dessous afin d'examiner la façon dont l'étrangeté est appréhendée par les différentes traductrices.

On constate ainsi que les trois traductions ont tendance à normaliser le texte et à rétablir sa syntaxe pour le rendre plus lisible même si les phrases tronquées en anglais sont par ailleurs parfaitement compréhensibles. Ce procédé est bien connu des traductologues, mais est aussi dénoncé par les théories féministes de la traduction qui privilégient plutôt le recours à un langage que l'on pourra identifier comme différent des normes établies.

Dans la traduction de 1976, une partie des phrases sans verbe se voit ainsi reconstituée, et à l'une des phrases tronquées sont ajoutés des points de suspension qui n'existent pas dans la version originale. La traduction de 2002 essaie par endroits de garder cette syntaxe tronquée (- seul, ce papier peint... - phrase par ailleurs mise en relief par sa séparation des autres paragraphes qui n'existe pas dans la version originale). Notons aussi que Diane de Margerie a également pris le parti de créer une phrase tronquée là où dans l'original ne se trouve qu'une simple exclamative (Tant il m'obsède - It dwells in my mind so!). Ces stratégies de compensation permettent au lecteur d'appréhender l'esprit du texte dans sa globalité.

À l'inverse, il apparaît dans la traduction de 1982 (qui montre aussi une tendance à lier les paragraphes) que la structure syntaxique des phrases sans verbe a été rétablie de manière quasi systématique. Le résultat est encore une fois une impression de fluidité qui ne se retrouve pas dans les deux autres traductions du texte. Cette recherche de fluidité entraîne parfois la traductrice à interpréter certaines phrases assez librement, comme en témoigne la traduction de Round and round and round - round and round and round - it makes me dizzy par Ces questions se répètent, se répètent et se répètent jusqu'à ce que la tête me tourne: explicitation particulièrement restrictive que le texte original ne permet pas de justifier.

Le tableau suivant récapitule les différents choix opérés par les traductrices: 
TABLEAU 1

La syntaxe défaillante dans les trois traductions de The Yellow Wallpaper

\section{The Yellow Wallpaper}

(1892)

One of those sprawling flamboyant patterns committing every artistic sin. (5)

But I don't mind it a bit - only the paper. (8)

It dwells in my mind so! (9)

I wonder how it was done and who did it, and what they did it for. Round and round and round - round and round and round - it makes me dizzy. (16)

A strip about as high as my head and half around the room. (18)
Le papier peint jaune (1976)

C'est un de ces motifs tape-à-l'œil et vulgaire [sic], un outrage à

l'harmonie, véritablement.

Mais tout m'indiffère. Seul ce papier peint...

Il tourne dans ma tête comme un vertige.

Je me demande

comment et par qui elle a été faite, et pourquoi. Elle tourne, tourne, tourne - elle tourne et tourne encore - elle me donne le vertige.

... : aussi haut que nous pouvions atteindre, et sur une surface qui couvrait la moitié de la pièce.
La chambre au papier peint (1982)

Les dessins en sont flamboyants et immenses, d'une laideur repoussante qui profane toute notion de beauté.

Pourtant cela ne me gêne Mais rien de tout cela ne pas du tout, seul le papier m'ennuie.

Il m’obsède tellement !

Je me demande

comment il a été fait, qui l'a fait et pourquoi. Ces questions se répètent, se répètent, et se répètent jusqu'à ce que la tête me tourne!

Nous avons découvert une partie du mur, à peu près de la hauteur de ma tête, tout autour de la moitié de la chambre.
La séquestrée (2002)

Son motif est vulgaire et voyant - une véritable injure à tout sens artistique.

me dérange - seul, ce papier peint...

Tant il m'obsède.

Je me demande qui l'a

faite, comment et pourquoi? Elle tourne autour de la pièce, tournoie - tournoie, tournoie, tournoie, j'en ai le vertige.

[dans le même paragraphe] - un long panneau à ma hauteur recouvrant la moitié de la chambre.

\subsection{Les oxymores et déclarations contradictoires}

L'étrangeté du texte tient aussi à quelques oxymores et déclarations contradictoires qui surviennent au début du journal intime de la narratrice. Ces oxymores reflètent bien évidemment les messages contradictoires qu'elle reçoit d'une part de son mari, autorité médicale, et d'autre part de son «bon sens», qu'elle se refuse à écouter sinon par l'entremise de la «femme dans le papier peint», seul déplacement qu'elle s'autorise: «So I will let it alone and talk about the house» (Gilman 2000: 4). Ces contradictions, au nombre de quatre, disparaissent à mesure que l'écriture du journal avance et que la narratrice s'enfonce dans son univers imaginaire.

Le premier oxymore se trouve dans une des phrases les plus souvent citées lors de l'analyse de la nouvelle. L'idée exprimée (l'amour peut-il se reconnaître dans le contrôle aveugle et exclusif de l'homme sur la femme?) se répète dans le deuxième exemple, et ces deux phrases sont traduites assez littéralement dans les trois versions françaises du texte, respectant les contradictions exprimées sur le plan sémantique, même si toutes ne gardent pas la conjonction de coordination (and dans la première phrase, because dans la deuxième) qui met en évidence l'oxymore dans chaque occurrence. La traduction de 1976 les remplace par des signes de ponctuation, mettant ainsi les deux propositions en parallèle, sans les lier. La traduction de 2002 conserve ces deux conjonctions, tandis que celle de 1982 a choisi de simplifier la première occurrence: 
TABLEAU 2

Les oxymores et déclarations contradictoires (1)

\section{The Yellow Wallpaper} (1892)

He is very careful and loving, and hardly lets me stir without special direction. (5)

It is so hard to talk with John about my case, because he is so wise, and because he loves me so. (12)
Le papier peint jaune (1976)

Il est très attentif et affectueux; je ne peux faire le moindre geste sans qu'il s'en inquiète.

C'est tellement difficile de parler avec John de mon cas, il est si sage, il m'aime tant.
La chambre au papier peint (1982)

Il est très attentif et très Il est très attentif, très tendre, il ne me laisse tendre, et me laisse à pas bouger sans s'occuper du moindre de donner ses instructions. mes mouvements.

Il est difficile de discuter C'est si difficile de de mon cas avec John discuter de mon cas avec parce qu'il est si lui, parce qu'il est si raisonnable et qu'il intelligent, et qu'il m'aime tant.

Il est intéressant par ailleurs de noter la différence de traduction du nom opposition entre le texte de 1976 (sollicitude) et ceux de 1982 (réprobation) et 2002 («opposition»). Il s'agit peut-être ici d'une erreur de traduction, mais il est également possible d'interpréter ce faux-sens comme une stratégie de compensation utilisant un léger oxymore (la sollicitude est une attention soutenue, mais dont l'affection n'est pas absente), absent du texte original, qui montrerait la sensibilité de la traductrice à la contradiction latente exprimée par la narratrice au fil du texte:

TABLEAU 3

Les oxymores et déclarations contradictoires (2)

The Yellow Wallpaper (1892)

I did write for a while in spite of them; but it does exhaust me a good deal

- having to be so sly about it, or else meet with heavy opposition. (4)

Le papier peint jaune (1976)

Pendant quelque temps, j'ai continué à écrire en dépit d'eux tous, mais cela m'épuise de devoir toujours feindre pour ne pas me laisser écraser par leur sollicitude.

\section{La chambre au papier peint (1982)}

La séquestrée (2002)

Pendant quelque temps, Malgré eux, j’ai quand j'ai écrit malgré eux, mais même réussi à écrire en fait, l'effort m'exténue pendant quelque temps, car je dois travailler furtivement, ou faire face à leur réprobation. mais il est vrai que cela m'épuise d'avoir à le faire si sournoisement, quand je n'ai pas à me heurter à leur pesante opposition.

En revanche, dans le dernier exemple, les trois traductions semblent ici faire un contresens sur lurid, adjectif signifiant blafard, mais aussi criard. La présence de l'adverbe yet liant les deux adjectifs indique une contradiction entre les deux termes qui n'est rendue dans aucune des trois traductions. Cette description étrange, terne et pourtant criard, devrait pourtant figurer dans la liste des oxymores présents dans le texte et qui sous-tendent le discours contradictoire de la narratrice, mais ce trait stylistique se retrouve entièrement gommé dans les trois versions de la langue cible: 
TABLEAU 4

Les oxymores et déclarations contradictoires (3)

The Yellow Wallpaper (1892)

It is a dull yet lurid orange in some places, a sickly sulphur tint in others. (6)
Le papier peint jaune (1976)

Par endroits, un orange déteint et blafard, ailleurs une teinte maladive et sulfureuse.
La chambre au papier peint (1982)

La séquestrée

(2002)

A certains endroits, il est Une couleur d'un orangé d'un orange terne et assourdi par endroits blafard; à d'autres, il et d'une teinte sulfureuse est d'une teinte sulfureuse et maladive.

\subsection{Les répétitions obsessionnelles de certains mots ou idées}

Enfin, au fur et à mesure que la nouvelle progresse, on remarque l'augmentation de répétitions quasi obsessionnelles qui font passer la narratrice de l'ambivalence à l'idée fixe. La répétition de moon, light et night au milieu de la nouvelle (p. 12-14) illustre en partie cette ambivalence, mais la récurrence la plus frappante est celle de creep (p. 8-20), terme décliné sous plusieurs formes grammaticales dans le dernier tiers du texte, et dont l'apparition régulière et pour le moins étrange donne lieu à plusieurs types de traduction.

Un des problèmes stylistiques posés par la traduction de la répétition de moon, light et night est la perte de l'allitération qui met en parallèle les deux derniers termes tout en les opposant. Dans la traduction de 1982, la traductrice a recours à l'allitération en «l» (à la lumière de la lune, les rayons illuminent...) et les deux traductrices des textes de 1982 et de 2002 utilisent des termes porteurs de la même étymologie (lumière/ illuminent» et éclat/éclaire). Par ailleurs, aucune des traductrices n’a conservé la répétition des mots composés de light qui apparaissent p. 13-14:

TABLEAU 5

les répétitions obsessionnelles (1)

\section{The Yellow Wallpaper} (1892)

It was moonlight. The moon shines in all around just as the sun does. (12)

By moonlight - the Par les nuits où la lune moon shines in all night ne laisse aucun répit when there is a moon - I dans la chambre, je wouldn't know it was the jurerais que ce n'est pas same paper.

At night in any kind of

light, in twilight, candlelight, lamplight, and worst of all by moonlight, it becomes bars! The outside pattern I mean, and the woman behind it is as plain as can be. (13-14)

\section{Le papier peint jaune} (1976)

C'était une nuit de lune. La lune éclaire toute la pièce comme un soleil. le même papier peint. Le soir tombé, le motif s'altère avec la lumière. Le crépuscule, la chandelle, la lampe, le clair de lune surtout, le déforment en une série de barreaux - le motif superficiel, je veux dire... La silhouette féminine se distingue alors parfaitement.

\section{La chambre au papier peint (1982)}

C'était un soir de clair de lune. L'astre d'argent brillait dans toutes les directions tout comme le le ferait un soleil. soleil.

A la lumière de la lune - les rayons illuminent la chambre toute la nuit quand il y a la lune - personne ne pourrait croire que c'est le même papier.

La nuit, sous n'importe quel angle, à la lumière d'une chandelle ou d'une de la lampe, et surtout lampe, et le pire de tout, de la lune, on croit voir au clair de lune, il se surgir des barreaux. transforme en barreaux. Je parle du motif au Je veux dire que le dessin premier plan. La femme extérieur, par rapport à la forme du dessous, se transforme en barreaux. [+Omission]
Dans l'éclat lunaire - quand elle est haute, la lune éclaire la pièce entière toute la nuit -, le papier devient méconnaissable. La nuit, peu importe l'éclairage, à la lumière du qui se cache derrière se distingue parfaitement. 
Considérons enfin creeping, qui représente de loin la répétition la plus flagrante du texte (on trouve vingt occurrences de creep et trois de crawl entre les pages 8 et 20). C’est également la récurrence la plus «étrangement familière», pour reprendre les termes de Freud, car l'idée de ramper, métaphore rendant compte de la condition de la narratrice, infantilisée et diminuée par son mari, renvoie également à l'instinct animal ou à l'insecte sur le mur (et donc sur le papier peint).

La plupart des occurrences de creep ne posent pas de problème de traduction. Le verbe équivalent, ramper, est donc utilisé dans les trois traductions (c'est surtout le cas dans les traductions de 1976 et 2002; la traduction de 1982, qui a recours à la traduction littérale à plusieurs reprises, privilégie l'équivalence dans toute la dernière partie du texte). Quant à crawl, quoique légèrement différent de creep, il est lui aussi systématiquement traduit en français dans les trois textes par ramper.

Le tableau suivant fait l'inventaire des occurrences de ces traductions littérales, dans lesquelles les choix traductionnels dans les trois versions sont assez homogènes ${ }^{9}$ :

\section{TABLEAU 6}

Les répétitions obsessionnelles (2)

\section{The Yellow Wallpaper (1892)}

Up and down and those absurd, unblinking rampent, et toujours ces eyes are everywhere. (8) yeux absurdes et fixes...

And it is like a woman stooping down and creeping about behind that pattern. I don't like it a bit. (11)

Sometimes I think there are a great many women behind [the pattern], and sometimes only one, and she crawls around fast, and her crawling shakes it all over. (16)

I think that woman gets out in the daytime! [...] It is the same woman, I know, for she is always creeping, and most women do not creep by daylight. (16)

II see her in that long shaded lane, creeping up and down. I see her in those dark grape arbors, creeping all around the garden.] que cette femme s'échappe pendant la journée!

[...] C'est la même femme, je le sais, parce qu'elle rampe. La plupart des femmes ne rampent pas dans la journée.

\section{Le papier peint jaune (1976)}

En haut, en bas, sur les
côtés, partout les têtes
rampent, et toujours ce
yeux absurdes et fixes..

On croit voir une femme accroupie et qui rampe au-delà du dessin.

Je n’aime pas ça.

Parfois je me dis qu'elles sont des multitudes,

parfois qu'elle est seule.

rampant à une vitesse

folle, ébranlant chaque motif.
Elle fait le tour en

\section{La chambre au papier peint (1982)}

Ils montent, et ils descendent, ils rampent $c$ d'un côté et de l'autre, ils ramper ces yeux sont partout, ces absurdes yeux qui ne clignotent jamais.

On dirait qu'il y a une femme qui se penche et se glisse derrière le dessin. Je n'aime pas cela du tout.

Quelquefois, j'imagine qu'il enferme un grand nombre de femmes, et d'autres fois une seule qui se déplace très vite et en rampant, produit toute allure, et qu'à force les secousses.

\section{La séquestrée} (2002)

[...]: en bas, en haut, de absurdes et fixes.

On dirait qu'une femme se penche jusqu'à terre pour aller ramper

derrière le dessin. Cela ne me plaît pas du tout. plusieurs femmes se dissimulent derrière le motif, et parfois qu'une de ramper à une telle
Parfois, il me semble que seule y rampe en rond, à
toute allure, et qu'à force vitesse le papier en est tout agité de secousses!

J'ai toute raison de croire Je crois que cette femme s'échappe pendant le jour! [...]

C'est de jour, je pense, que cette femme s'évade. [...]

C'est toujours la même Il s'agit de la même femme, je le sais, car elle femme, j'en suis sûre, car rampe toujours, et la elle ne cesse de ramper plupart des femmes ne le et, d'habitude, les font pas en plein jour. femmes ne rampent jamais à la lumière du jour.

[omission] 
I see her on that long road under the trees, creeping along, and when a carriage comes she hides under the blackberry vines.
Je la vois, là, rampant Je la vois se traîner sur dans la longue allée sous ce long chemin sous les les arbres. Elle se cache arbres, et quand une dans les haies de ronces quand passe une voiture. Je ne la blâme pas le

I don't blame her a bit. It moins du monde! Ce must be very humiliating doit être très humiliant to be caught creeping by d'être surprise à ramper daylight! I always lock the door when I creep by daylight. I can't do it at night, for I know John would suspect something at once. (16-17) cache sous les mûriers sauvages.

Je la comprends parfaitement. Ca doit être si humiliant d'être surprise à ramper en plein jour. nuit, car John se en plein jour!

Je ferme toujours la porte avant de me mettre à ramper dans la journée. La nuit il n'en est pas question: John se douterait immédiatement de quelque chose. sur la longue route

voiture s'approche, elle se ombragée, se cachant

That was clever, for really Belle réponse en vérité, I wasn't alone a bit! As j'étais loin d'être seule! soon as it was moonlight La clarté de la lune avait and that poor thing began to crawl and shake the pattern, I got up and ran to help her.

à peine pénétré dans la
chambre que cette
pauvre créature
pauvre créature commençait à ramper et à secouer ses grilles, je me suis levée et me suis précipitée vers elle pour
Je ferme toujours la porte de ramper ainsi en plein à clé quand je rampe jour! dans la journée. Moi, une fois le jour Impossible de le faire la levé, je m’enferme toujours à clef quand je rampe. Il m'est impossible de le faire la nuit - cela éveillerait les soupçons de John. chose.

derrière les buissons de voiture

Ce n'est pas moi qui la blâmerais. Quelle humiliation ce doit être d'être surprise en train

C'était très astucieux, car Quelle réponse rusée, car en réalité je n'étais pas je ne suis pas seule du du tout seule! Dès qu'il y tout! a eu le clair de lune, et A peine la lune s'est-elle que la pauvre créature mise à briller, et cette s'est mise à ramper et à malheureuse créature à ramper, que je me suis levée pour l'aider. l'aider.

Il existe cependant quelques situations où n'a pas été fait le choix de la traduction littérale (qui aurait pu servir l'écriture de la folie) : creep, terme polysémique, sera donc traduit par des termes différents dans les trois traductions. The moon creeps... devient ainsi glisse / se faufile / rôde; "The smell creeps" se transforme en se répand / se glisse / se traîne (voir tableau):

\section{TABLEAU 7}

\section{Les répétitions obsessionnelles (3)}

\section{The Yellow Wallpaper} (1892)

[...] I hate to see it [the moon] sometimes, it creeps so slowly, and always comes in by one window or another. (12)

But there is something else about that paper the smell! [...]

It creeps all over the house. (15)

\section{Le papier peint jaune} (1976)

[...] Parfois je la hais. Elle glisse si lentement, elle s'insinue d'une fenêtre à l'autre.

Autre chose aussi au sujet de ce papier peint - l'odeur! [...]

Elle se répand dans toute la maison.

\section{La chambre au papier} peint (1982)

[...] Je déteste quelquefois voir la lune; elle se faufile si lentement, et elle pénètre toujours par une fenêtre ou l'autre.

Mais ce papier a une autre caractéristique son odeur! [...]

Elle se glisse partout, partout dans la maison.

\section{La séquestrée}

(2002)

\section{[...] Parfois je déteste la} regarder: elle rôde si furtivement, elle se glisse toujours à travers une fenêtre ou une autre.

Et puis il y a autre chose encore le concernant: son odeur! [...]

Elle se traîne dans toute la maison. 
La polysémie difficile à rendre de creepy (until I felt creepy p. 12) n’a été conservée dans aucune des traductions. Dans chaque cas, les trois traductrices ont recours à l'équivalence ou à l'explicitation. Le choix du texte de 2002 nous paraît cependant judicieux car il file la métaphore de la lassitude que l'on retrouve chez la narratrice tout au long du texte:

TABLEAU 8

Les répétitions obsessionnelles (4)

The Yellow Wallpaper (1892)

John was asleep, and I hated to waken him, so I kept still and watched the moonlight on that undulating wall-paper till I felt creepy. (12)

\section{Le papier peint jaune} (1976)

John dormait, je n'aurais pas supporté de le réveiller, aussi suis-je restée immobile, à regarder s'étirer cette clarté ondoyant sur le papier peint - jusqu'à en avoir la chair de poule.
La chambre au papier
peint (1982)

John dormait, et comme je ne voulais pas le réveiller, je suis donc restée silencieuse à regarder le clair de lune sur ce papier onduleux jusqu'à ce que j'en éprouve un sentiment étrange.

\section{La séquestrée} (2002)

John était endormi et je répugnais à le réveiller, alors je suis restée immobile à regarder le clair de lune bouger sur le papier peint jusqu'à ce que j'en éprouve des frissons.

Il y a par contre des cas où la traduction de creep ne pose pas de problème, et pour lesquels les trois textes français opèrent des choix de traduction différents, notables en particulier à la fin du texte, où le mot fait référence soit à la femme dans le papier peint, soit à la narratrice elle-même. Les deux traductions de 1976 et 2002, comme dans les premiers exemples, font le choix du verbe ramper et de sa répétition, mais le texte de 1982 opte le plus souvent pour des expressions de substitution: se faufiler, se glisser, se traîner, avancer ou passer sur son corps comme le montre le tableau suivant:

TABLEAU 9

Les répétitions obsessionnelles (5)

\section{The Yellow Wallpaper (1892) \\ $[\ldots]$ \\ Le papier peint jaune La chambre au papier (1976)} And though I always see Et même si je pouvais ne Et bien que je la voie her, she may be able to pas la quitter des yeux, toujours, elle pourrait creep faster than I can elle serait peut-être turn.

I have watched her sometimes away off in the open country, creeping as fast as a cloud shadow in a high wind. (16-17) capable de ramper encore plus vite avant que j'aie le temps de me retourner!

Je l'ai aperçue parfois, en pleine campagne, ramper à vive allure, comme l'ombre d'un nuage chassé par la bourrasque.

\section{être capable de se} traîner plus vite que je ne peux me retourner! Je l'ai aperçue quelquefois qui s'éloignait en rase mpagne, elle avançait grande distance, aussi rapidement que progressant à travers la l'ombre d'un nuage campagne, rampant, poussé par un vent violent.

\section{La séquestrée (2002)}

[...] Et même si je ne cesse de l'observer, elle est sans doute capable de ramper encore plus vite, quand bien même j'aurais eu le temps de me retourner. Parfois, j'ai pu l'apercevoir à une rampant, plus rapide que l'ombre d'un nuage filant dans le grand vent. 
I don't like to look at the J'en suis arrivée à ne windows even - there are too many of those creeping women, and they creep so fast. I wonder if they all come out of the wall-paper as I did. [...] It is so pleasant to be out in this great room and creep around as I please!

I don't want to go outside. I won't, even if C'est si agréable d'être Jennie asks me to. For outside you have to creep on the ground, and everything is green instead of yellow.

But here I can creep smoothly on the floor, and my shoulder just fits in that long smooch around the wall, so I cannot lose my way. (19) jaun

plus aimer regarder par la fenêtre. Ces femmes qui rampent sont partout, et elles rampent si vite...

Je me demande si, comme moi, elles se sont toutes échappées de ce papier peint.

\section{[...}

C'est si agréable d'êt
là, libre, dans cette grande chambre et de ramper quand bon me semble!

Je ne veux pas sortir. Je ne le veux pas, même si Jennie me le demande! Parce que dehors il faut ramper à même le sol, où tout est vert et non aune.

Mais ici, je peux ramper sans heurt sur le parquet. L'épaule bien coincée dans le sillon du mur, je ne peux pas perdre mon chemin.

Je n'aime même plus Je n'ai même plus envie hegard hors de regarder par la des fenêtres, tant il y a fenêtre - il y a tant de de femmes qui s'y traînent, et à quelle vitesse!

Je me demande si elles sont toutes sorties de ce papier, comme je l'ai

fait, moi.

[...]

C'est si agréable d'être

dehors dans cette grande pièce et de ramper autant que je veux!

Je ne veux pas aller dehors. Je ne le ferais pas, même si Jennie me le demandait.

Parce que, hors de la maison, il faut se traîner par terre, et tout est vert au lieu de jaune. ces femmes qui rampent, qui rampent à une de ces allures! Je me demande si elles sont toutes, comme moi, sorties du papier peint?

[sic]

[...]

C'est tellement agréable d'être ici, dans cette chambre spacieuse, et d'y ramper en toute liberté.

Je refuse de sortir. Je m'y opposerai, même si Jennie me le demande. Car dehors, il faut ramper sur le sol inégal et tout est vert au lieu d'être jaune.

Mais ici je peux ramper à mon aise sur le Ici, au contraire, je peux parquet, et comme mon me glisser tout doucement sur le épaule peut se loger dans la longue faille plancher, et mon épaule creusée autour de la s'ajuste parfaitement à pièce, je ne risque pas de cette longue trace me perdre. autour du mur, de sorte que je ne peux pas m'égarer.

"What is the matter?" he cried. "For God's sake, what are you doing?"

I kept on creeping just the same, but I looked at him over my

shoulder.

"I've got out at last," said I, "in spite of you and Jane. And I've pulled off most of the paper, so you can't put me back!"

Now why should that man have fainted? But he did, and right across my path by the wall, so that I had to creep over him every time. (20)
«Que se passe-t-il» a-t-il «Qu'est-ce qu'il y a? crié. «Pour l'amour de s'est-il exclamé. Pour Dieu, que fais-tu?» l'amour de Dieu, que Je continuai à ramper fais-tu?»

comme si de rien J'ai continué à me n'était, non sans lui avoir lancé un regard par-dessus mon épaule.

«J'en suis venue à bout finalement, ai-je dit, en dépit de toi et Jane. J'ai arraché presque tout le papier peint, et tu ne risques pas de m'y renfermer!»

\section{traîner quand même,} mais je l'ai regardé

- Pour l'amour de Dieu! a-t-il hurlé. Que se passe-t-il? Qu'est-ce que tu fais?

J'ai continué de ramper comme si de rien n'était, le regardant par-dessus mon épaule. par-dessus mon épaule. "Je suis enfin libérée, - Je suis enfin délivrée, ai-je dit, malgré Jane et dis-je. Malgré toi, toi. Et j'ai arraché malgré Jennie. Et j’ai presque tout le papier, arraché presque tout le donc tu ne peux pas m'y papier. Vous ne pourrez remettre!»

Quelle raison cet homme aurait-il de plus me séquestrer! Je vous le demande: pourquoi donc cet s'évanouir? Et pourtant homme s'est-il évanoui? Pourquoi a-t-il fallu que c'est ce qui s'est produit. Pourtant, c'est ce qu'il a cet homme Il s'est affalé en travers s'évanouisse? du chemin que je

Car il s'est évanoui, et il m’étais tracé le long du est tombé juste en travers de mon chemin, près du mur. mur, de sorte que je fus obligée de passer sur son corps chaque fois que je fis le tour de la fait, juste en travers de mon chemin, près du mur, si bien que chaque fois, il me faudra ramper par-dessus son corps...
Et chaque fois, il me que je fis l
faut ramper par-dessus chambre!

son corps! 
Ce tableau fait apparaître plus qu'ailleurs la différence fondamentale entre les deux textes de 1976 et 2002 et le texte de 1982, ce dernier tendant à gommer nombre de répétitions pour les remplacer par des termes plus ou moins équivalents. Si l'on considère la cohérence interne des trois textes, on remarque ainsi que la traductrice de l'édition québécoise semble effacer systématiquement des répétitions peut-être jugées maladroites. En optant pour la fluidité, elle gomme cependant ce trait stylistique caractéristique du texte original qui insistait sur l'obsession de la narratrice.

À la fin du récit, une différence majeure apparaît entre les trois traductions, qui symbolise peut-être la tendance que chacune exprime. Le retournement de situation décrit dans le dernier paragraphe est bien conservé dans les trois cas (renversement des rôles entre la narratrice et son époux, qui se trouve ici «féminisé» par son évanouissement - pathologie typiquement féminine -, et disparition du nom de ce dernier - cet homme), mais les deux traductions qui ont choisi de préserver l'étrange de creep (Et chaque fois il me faut ramper par-dessus son corps, 1976; si bien que chaque fois il me faudra ramper par-dessus son corps, 2002) préservent et soulignent un élément essentiel de la nouvelle - et un élément fondamental du discours critique sur la nouvelle: l'ambiguïté du triomphe de la narratrice, qui passe sur le corps de son mari, certes, mais en rampant. On peut ainsi se demander si la dernière phrase de la traduction de 1982, en perdant cette image, ne perd pas non plus son impact et n'est pas à la limite du contresens. La neutralisation plus générale de l'étrange dans la traduction de cette nouvelle semble donc contreproductive d'un point de vue purement littéraire, car elle perd sa richesse polysémique. L'étrange, outre son expression par la préservation d'éléments aussi divers que la mise en page du texte, la syntaxe défaillante, les oxymores et déclarations contradictoires ou les répétitions obsessionnelles, naît donc aussi en grande partie de la préservation de l'ambiguité qu'exprime le texte.

Pour terminer, notons que l'engagement féministe des deux traductrices de 1976 et 2002 s'exprime aussi par le changement de temps d'un simple verbe: alors que la traductrice de l'édition de 1982 conserve la forme passée de la dernière phrase (I had to creep devient je fus obligée... et clôture donc le récit), les deux autres traductrices font un choix plus radical. Le présent de il me faut ramper (1976) confère au récit un caractère universel, tandis que le futur exprimé par il me faudra ramper (2002) semble souligner le caractère inéluctable et implacable de l'oppression masculine qu'entend exprimer Gilman.

\section{Conclusion}

Cette analyse comparative des différences de traduction entre chaque texte confirme donc les tendances stylistiques générales identifiées en première lecture. Nous pouvons ainsi avancer que la traduction publiée au Québec en 1982 se démarque nettement des deux traductions françaises de 1976 et 2002 dans sa manière de gommer l'étrangeté de la nouvelle pour lui substituer une fluidité de lecture et une lisibilité plus importantes que les autres traductions. En réduisant le nombre de répétitions, en effaçant les ruptures syntaxiques et typographiques, la traductrice atténue l'étrange, et par là même le discours de la folie, qui est mieux préservé par ailleurs dans les deux autres traductions. La traduction de 1982, de par son approche cibliste, apparaît ainsi, et peut-être plus que celle de 1976, comme un exemple type de première 
traduction que l'on pourrait qualifier de traduction de vulgarisation. Lancrage de cette dernière dans un paratexte féministe (la postface d'Elaine R. Hedges) semble finalement indépendant de l'approche du texte lui-même: cette vulgarisation qui, dans les premières traductions, semble nécessaire à l'accession au sens se trouve en effet critiquée par des théoriciennes telles que Gayatri Spivak (2000) qui les trouvent contreproductives dans le sens où elles atténuent la force du discours féministe. La traduction des éditions Des femmes, quant à elle, semble d'une part plus fidèle à la lettre qu'à l'esprit et, d'autre part, en n'offrant pas de paratexte didactique particulier à la nouvelle, laisse le lecteur appréhender le texte traduit en mentionnant seulement qu'il s'agit là d'un texte féministe.

La retraduction de 2002, quant à elle, semble répondre aux caractéristiques énoncées par Berman et Bensimon, et met en valeur les traits stylistiques de l'original qui font de lui un "texte étrange» : conservation de certains archaïsmes, respect de la structure des propositions imparfaites et nombreuses stratégies de compensation afin de contrebalancer les occurrences où la traduction littérale s'avère malgré tout impossible. L'exemple de la traduction par Diane de Margerie de Wharf (7) par môle (20) (n. m.: embarcadère, mais n. f.: croissance anormale du placenta) témoigne de sa volonté de conserver à la fois l'étrange grâce à un choix de termes peu familiers, et d'ancrer un sous-texte directement en rapport avec la diégèse et son discours féministe. On peut considérer qu'il s'agit là d'un cas d'intervention clairement identifiable de la part de la traductrice féministe, qui offre ainsi à ses lecteurs un texte qui en fin de compte va se trouver en contradiction avec la note de l'éditeur le présentant comme un livre dont l'enjeu généraliste est de répondre à «cette forme d'incarcération à quoi menace de se résoudre toute vie si nous lui refusons les moyens de l'indispensable effraction» (Gilman 2002: 10). On semble donc assister ici à un mouvement contradictoire dans lequel la traduction et son paratexte fonctionnent indépendamment l'un de l'autre.

Si dans tous les cas la médiation du paratexte est importante pour l'accès au texte étrange, le discours féministe présent dans chacune des traductions dépend finalement dans une large mesure de la manière dont le discours de la folie sera traité d'un point de vue stylistique, et plus précisément de la manière dont le respect de la lettre assurera le respect de la complexité nécessaire à l'appréhension d'un message qui signifie en lui-même autant que par lui-même, et qui porte ainsi en lui l'étrangeté de son origine.

\section{NOTES}

1. La nouvelle est parue sous son nom d'épouse. Stetson, Charlotte Perkins (1892): The Yellow Wallpaper. A Story. The New England Magazine. 11(5):647-657. Consultée le 3 novembre 2011, $<$ http://digital.library.cornell.edu/cgi/t/text/pageviewer-idx?c=newe;cc=newe;rgn=full\%20text; idno=newe0011-5; didno=newe0011-5; view=image;seq=0655;node=newe0011-5\%3A12 $>$. L'édition utilisée dans la présente étude est la suivante: Gilman, Charlotte Perkins (1892/2000): The Yellow Wallpaper and Other Writings. New York: The Modern Library.

2. Les rééditions suivantes de la nouvelle, répertoriées par Catherine Golden, rendent bien compte de cet état de fait: The Great Modern American Stories (1920), The New York Evening Post (1922), American Mystery Stories (1927), The Golden Book Magazine (1933), The Haunted Omnibus (1937), The Midnight Reader: Great Stories of Haunting and Horror (1942), About Women: A collection of Short Stories (1943), Ghostly Tales to be Told (1950), More Macabre (1960), A Chamber of Horrors: An Anthology of the Macabre in Words and Pictures (1965), Psychopathology and Literature (1966), Eight Strange Tales (1972), et The Oven Birds: American Women on Womanhood (1972). Suivront 
des rééditions dans Wolf's Complete Book of Terror (1979), et dans Haunted Women: The Best Supernatural Tales by American Women Writers (1985) et The Norton Anthology of Literature by Women (1985). Voir la chronologie complète des publications de Gilman dans l'ouvrage de Golden: Golden, Catherine (2004): Charlotte Perkins Gilman's The Yellow Wallpaper: A Sourcebook and Critical Edition. New York/London: Routledge, 17-25. C'est aussi dans ce contexte que The Yellow Wallpaper fait l'objet, aux États-Unis, d'adaptations radiophoniques, dont celle produite par CBS en 1948 pour le compte d'une série d'émissions intitulée Suspense. La réécriture, le jeu des acteurs et l'ajout d'une partition musicale angoissante contribuent à mettre l'accent presque exclusivement sur le caractère fantastique du récit et relègue au second plan la dimension psychologique du personnage tout comme le commentaire social de l'auteure. (Je remercie M. Christopher Pinon, qui a porté cette information à ma connaissance).

3. Gilman, Charlotte Perkins (1892/1976): Le papier peint jaune. (Traduit de l'anglais par le collectif de traduction des éditions Des femmes) Paris: Des femmes. Réédité en 2007.

4. Gilman, Charlotte Perkins (1892/1982): La chambre au papier peint. (Traduit de l'américain par Mary M. Millman) Sherbrooke: Éditions Naaman.

5. Gilman, Charlotte Perkins (1892/2002): La séquestrée. (Traduit de l’anglais par Diane de Margerie) Paris: Éditions Phébus. Réédité en 2008.

6. Aucun nom spécifique de traductrice n'apparaît dans les traductions de ce collectif - choix intéressant et paradoxal dans la mesure où la traduction féministe à partir des années $1970 \mathrm{a}$ justement tenté de réimposer la personne du traducteur en tant qu'auteur à part entière.

7. Fouque, Antoinette (s.d.): Les éditions Des femmes. Espace des femmes. Consultée le 4 mai 2011, $<$ http://www.desfemmes.fr/histoire.htm >. Au 3 novembre 2011, le site est indisponible, mais la présentation d'A. Fouque peut être lue sur le blogue de G. Depis:

Depis, Guilaine (s.d.): Éditions Des femmes - Blog de Guilaine Depis, ancienne attachée de presse (avril 2007-juillet 2010). Consultée le 3 novembre 2011, <http://editionsdesfemmes.blogspirit.com/ about.html>.

8. "Ce récit est un des rares témoignages littéraires d'une femme du dix-neuvième siècle face à la politique sexuelle entre homme et femme, entre époux et épouse; une histoire d'aberration mentale, mais aussi un document féministe qui remonte à un temps où peu d'écrivains se sentaient libres d'aborder un tel sujet» (Millman, Mary M. 1982: quatrième de couverture; voir note 4 pour la référence complète).

9. Notons également l'omission de la phrase en italique et entre crochets. Comme elle se répète dans les trois traductions, nous supposons qu'elle ne se trouvait pas dans l'édition originale utilisée par les traductrices.

\section{RÉFÉRENCES}

Bensimon, Paul (1990): Présentation. Palimpsestes. 4:ix-xiii.

Berman, Antoine (1990): La retraduction comme espace de la traduction. Palimpsestes. 4:1-7.

Genette, Gérard (1982): Palimpsestes, la littérature au second degré. Paris: Éditions du Seuil.

Gilman, Charlotte Perkins (1913): Why I wrote The Yellow Wallpaper. Forerunner. 4:213.

Lefevere, André (1992): Translation, Rewriting, and the Manipulation of Literary Fame. London: Routledge.

Simon, Sherry (1996): Gender in Translation: Cultural Identity and the Politics of Transmission. New York: Routledge.

Spivak, Gayatri (2000): The Politics of Translation. In: Lawrence Venuti, dir. The Translation Studies Reader. New York/London: Routledge, 397-416.

Von Flotow, Luise (1991): Feminist Translation: Contexts, Practices and Theories. TTR. 4(2):6984. 